\title{
Health Care Costs of Anal Cancer in a Commercially Insured Population in the United States
}

\author{
Chi-Fang Wu, MS; Li Xu, PhD; Shuangshuang Fu, MS; Ho-Lan Peng, PhD;
} Craig A. Messick, MD, FACS, FASCRS; and David R. Lairson, PhD

\begin{abstract}
BACKGROUND: The incidence and death rate of anal cancer in the United States has been increasing on average $2 \%-3 \%$ per year over the past 10 years. Human papillomavirus (HPV) vaccination is a potentially viable prevention strategy, since about $80 \%$ of anal cancers are attributable to HPV. To understand the effect of HPV vaccination, it is important to estimate the treatment costs for the HPV-related disease.
\end{abstract}

OBJECTIVE: To estimate the 2-year per patient mean direct health care costs associated with anal cancer in a commercially insured population in the United States.

METHODS: All newly diagnosed anal cancer patients were identified from a 2011-2014 Truven MarketScan database. Matched population controls were selected from the database with a 2-step matching method using demographic, comorbidity, and health care cost variables. Costs for the first 2 years were measured for cancer patients and controls. The difference in costs between the groups was calculated to estimate the costs associated with anal cancer treatment. A generalized linear model with gamma distribution and log link function was applied to estimate the costs for censored months for the patients who did not have at least 2 years of follow-up.

RESULTS: 1,976 patients with anal cancer and 1,976 controls were included in the study. The first 2-year per patient adjusted mean cost associated with anal cancer treatment was $\$ 127,531(S D=\$ 189,064)$. Male sex, cancer diagnosis, higher Charlson Comorbidity Index score, and higher prediagnosis costs were significantly associated with higher monthly costs. Higher psychiatric diagnostic group scores were associated with lower monthly costs. Anal cancer treatment costs were highest in the first 6 months after diagnosis (per patient per month [PPPM] mean $=\$ 12,846$ ), leveling off at a much lower monthly cost during the subsequent 18 months of the 2-year period (PPPM mean $=\$ 3,717$ ).

CONCLUSIONS: The first 2-year costs associated with anal cancer treatment were substantial. Given that approximately $80 \%$ of anal cancers are attributable to HPV infection, this study provides important parameters for estimating the potential economic benefit of HPV vaccination.

J Manag Care Spec Pharm. 2018;24(11):1156-64

Copyright $($ 2018, Academy of Managed Care Pharmacy. All rights reserved.

\section{What is already known about this subject}

The literature on anal cancer treatment cost for the privately insured population in the United States is limited.

The average lifetime cost per patient with anal cancer has been estimated at \$51,200 among a Medicare-insured population.

The estimation for a privately insured population is important for evaluating the economics of immunization strategies.

\section{What this study adds}

This study used real-world U.S. private insurance claims payment data to estimate the first 2-year per patient mean treatment cost for anal cancer cases.

Study results showed that the first 2-year per patient mean cost associated with anal cancer treatment was $\$ 127,531$

A nal cancer is rare, accounting for $0.5 \%$ of all new cancer cases in the United States. In 2017, there were 8,200 new cases of anal cancer diagnosed in the United States, and it was estimated that 1,100 people would die from this cancer. ${ }^{1}$ The incidence of anal cancer has more than doubled since 1975 and has been increasing on average $2.2 \%$ each year over the past 10 years. ${ }^{2}$ The death rate of anal cancer has been increasing on average $3.2 \%$ each year over the past 10 years, with no improvement in survival over the past 4 decades. ${ }^{2}$ Anal cancer affects more women than men, showing a gender ratio of 1.4 to 1 in the U.S. population. Most cases are diagnosed in individuals aged 50 years or older. ${ }^{2}$ Almost all anal cancers are attributable to infection with oncogenic types of human papillomavirus (HPV). In a study of anal squamous cell carcinomas diagnosed between 1993 and 2005, HPV deoxyribonucleic acid was detected in $91 \%$ of anal cancers, and in $79 \%$ of those cases, the HPV type was 16 or $18 .{ }^{3}$ Human immunodeficiency virus (HIV) infection is another risk factor associated with anal cancer occurrence, but it is unclear whether HIV infection directly causes anal carcinogenesis or if its effect is mediated through HPV. ${ }^{4}$ There is a markedly high incidence of anal cancer in HIV-positive men who have sex with men, and a meta-analysis of 21 studies showed that the prevalence of anal HPV infection in HIV-positive men who had sex with men was $93 \% .^{5}$

Cost-effectiveness studies provide information about the potential efficiency of using pharmacists and/or other alternative settings for increasing the HPV vaccination rate. ${ }^{6}$ However, cost-effectiveness studies are limited by a lack of current real-world estimates of treatment costs for HPV-related cancers. For example, leading U.S. economic analyses are based on a study by $\mathrm{Hu}$ and Goldie (2008) that approximated the lifetime costs of anal cancer from a study of rectal cancer in a Canadian population, ${ }^{7-9}$ and the leading U.S. HPV economic 


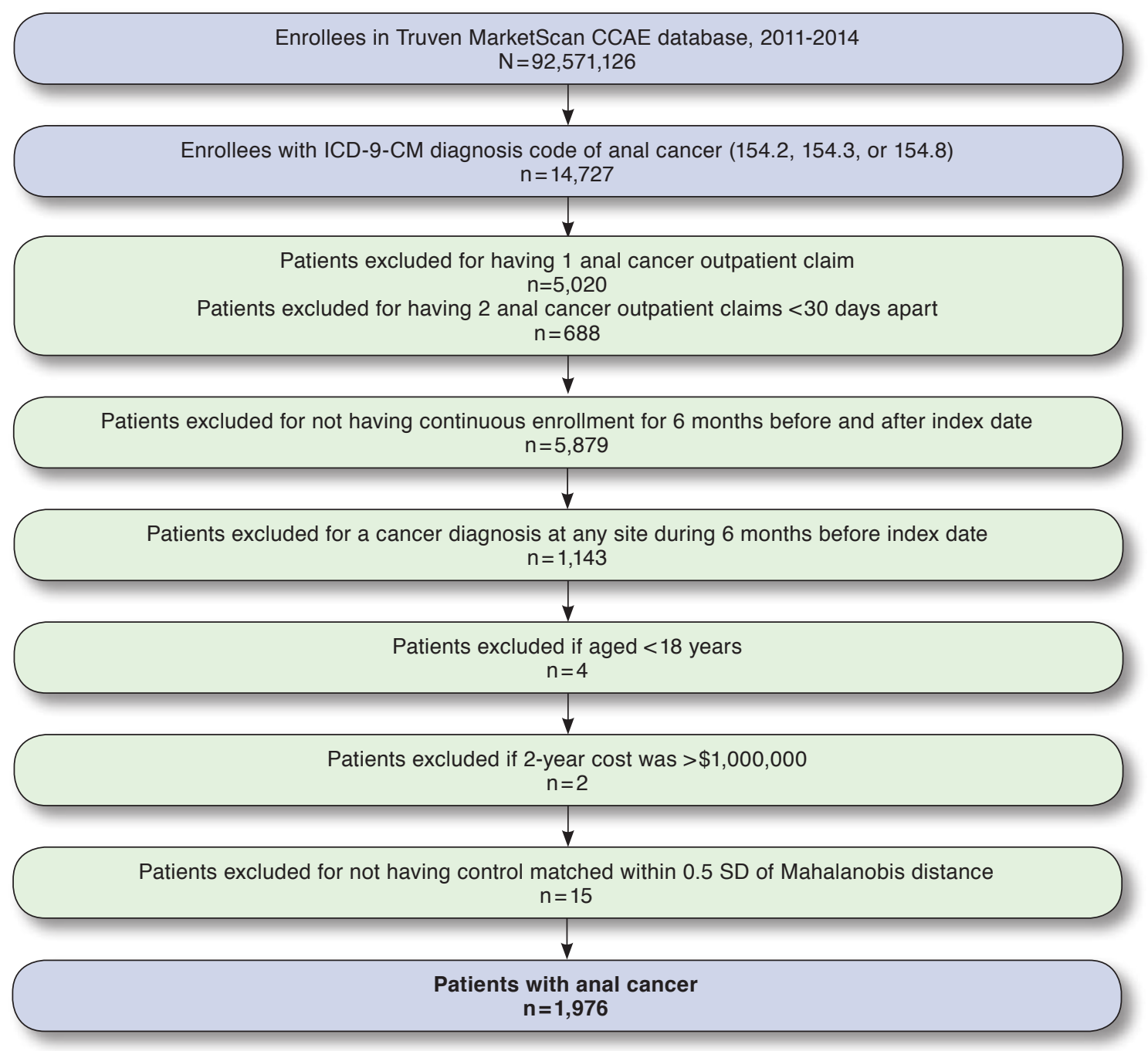

CCAE = Commercial Claims and Encounters; ICD-9-CM=International Classification of Disease, Ninth Revision, Clinical Modification; SD = standard deviation

models were validated by a study of the costs of colorectal cancer. ${ }^{9-11}$ A recent U.S. study of the costs of treating anal cancer was based on Surveillance, Epidemiology and End Results (SEER)-Medicare data and, therefore, covered only persons aged 65 years and older. ${ }^{12}$ A study by Olsen et al. (2012) provided estimates for the first 3 years of hospital costs in Denmark for the treatment of anal cancer. ${ }^{13}$ However, the findings of this study are not directly applicable to the United States given the differences between the 2 countries in populations, cost structure, and treatment protocols.

The purpose of this study was to estimate the per patient treatment costs over the first 2 years after diagnosis associated with incident cases of anal cancer from a large U.S. database of commercial insurance claims. Current real-world cost estimates will serve as an important input to decision models that inform policymakers about the potential cost offsets associated with expansion of U.S. HPV immunization rates, which are low compared with other developed countries and the Healthy People 2020 goal of $80 \%$ coverage. ${ }^{14}$ For U.S. adolescents aged 13-17 years, approximately $42 \%$ of females and $28 \%$ of males completed the HPV vaccination series of 3 doses. ${ }^{15,16}$

\section{Methods}

\section{Data Source and Study Population}

Costs were estimated with the Truven MarketScan 20112014 Commercial Claims and Encounter (CCAE) database, a large health insurance database that contains disease codes, procedure codes, service codes, and payments for individual 


\begin{tabular}{|c|c|c|c|}
\hline Characteristic & $\begin{array}{l}\text { Case Group } \\
(\mathrm{n}=1,976)\end{array}$ & $\begin{array}{c}\text { Control Group } \\
(n=1,976)\end{array}$ & $P$ Value \\
\hline Age, years, mean (SD) & $54.37 \quad(7.00)$ & $54.02 \quad(7.23)$ & 0.124 \\
\hline $\begin{array}{l}\text { Enrollment duration, } \\
\text { months, mean (SD) }\end{array}$ & $19.85(10.16)$ & $20.94(10.57)$ & $<0.001$ \\
\hline \multicolumn{3}{|l|}{ Sex } & Matched \\
\hline Male & $715(36.18)$ & $715(36.18)$ & \\
\hline Female & $1,261 \quad(63.82)$ & $1,261 \quad(63.82)$ & \\
\hline \multicolumn{3}{|l|}{ Duration of follow-up } & 0.054 \\
\hline Less than or equal to 1 year & $602(30.47)$ & $547(27.68)$ & \\
\hline More than 1 year & $1,374 \quad(69.53)$ & $1,429(72.32)$ & \\
\hline \multicolumn{3}{|l|}{ Employment classification } & 0.126 \\
\hline Salaried & $291(14.73)$ & $340 \quad(17.21)$ & \\
\hline Hourly & $349(17.66)$ & $333(16.85)$ & \\
\hline Other & $235(11.89)$ & $250(12.65)$ & \\
\hline Missing/unknown & $1,101 \quad(55.72)$ & $1,053(53.29)$ & \\
\hline \multicolumn{3}{|l|}{ Employment status } & $<0.001$ \\
\hline Active full time & $736(37.25)$ & $920(46.56)$ & \\
\hline Active part time or seasonal & $14 \quad(0.71)$ & $15 \quad(0.76)$ & \\
\hline Retired & $252(12.75)$ & $197 \quad(9.97)$ & \\
\hline $\begin{array}{l}\text { COBRA beneficiary (con- } \\
\text { tinue)/long-term disability/ } \\
\text { surviving spouse/dependent }\end{array}$ & $30 \quad(1.52)$ & $12 \quad(0.61)$ & \\
\hline Other/unknown & $944(47.77)$ & $832(42.11)$ & \\
\hline \multicolumn{3}{|l|}{ Union membership } & 0.468 \\
\hline Union member & $228(11.54)$ & $245(12.40)$ & \\
\hline Nonunion member & $559(28.29)$ & $581(29.40)$ & \\
\hline Other & $88 \quad(4.45)$ & $97 \quad(4.91)$ & \\
\hline Missing/unknown & $1,101 \quad(55.72)$ & $1,053(53.29)$ & \\
\hline \multicolumn{3}{|l|}{ Health insurance plan type } & 0.528 \\
\hline Comprehensive & $66 \quad(3.34)$ & $67 \quad(3.39)$ & \\
\hline EPO/unknown & $91 \quad(4.61)$ & $84 \quad(4.25)$ & \\
\hline $\begin{array}{l}\mathrm{HMO} / \mathrm{POS} \text { with capitation/ } \\
\mathrm{POS}\end{array}$ & $384(19.43)$ & $388(19.64)$ & \\
\hline $\mathrm{PPO}$ & $1,261 \quad(63.82)$ & $1,232(62.35)$ & \\
\hline CDHP/HDHP & $174 \quad(8.81)$ & $205(10.37)$ & \\
\hline \multicolumn{3}{|l|}{ Geographic area (division) } & Matched \\
\hline New England & $106 \quad(5.36)$ & $106 \quad(5.36)$ & \\
\hline Middle Atlantic & $300(15.18)$ & $300(15.18)$ & \\
\hline East North Central & $339(17.16)$ & $339(17.16)$ & \\
\hline West North Central & $93 \quad(4.71)$ & $93 \quad(4.71)$ & \\
\hline South Atlantic & $398(20.14)$ & $398(20.14)$ & \\
\hline East South Central & $126 \quad(6.38)$ & $126 \quad(6.38)$ & \\
\hline West South Central & $227(11.49)$ & $227(11.49)$ & \\
\hline Mountain & $103 \quad(5.21)$ & $103 \quad(5.21)$ & \\
\hline Pacific/missing & $284(14.37)$ & $284(14.37)$ & \\
\hline $\begin{array}{l}\text { Charlson Comorbidity Index } \\
\text { score, mean (SD) }\end{array}$ & $0.04 \quad(0.32)$ & $0.04 \quad(0.38)$ & 0.855 \\
\hline $\begin{array}{l}\text { Psychiatric diagnostic groups } \\
\text { scores, mean (SD) }\end{array}$ & $0.01 \quad(0.10)$ & $0.01 \quad(0.09)$ & 0.738 \\
\hline $\begin{array}{l}\text { Prediagnosis costs, } \\
\text { mean (SD), U.S. dollars }\end{array}$ & $2,653(7,712)$ & $1,902 \quad(5,219)$ & $-0.100^{b}$ \\
\hline
\end{tabular}

aValues in table are number of patients (percentage) unless otherwise indicated. ${ }^{b}$ Applied standardized difference for comparison.

$C D H P=$ consumer-driven health plan; $C O B R A=$ Consolidated Omnibus Budget Reconciliation Act; $\mathrm{EPO}=$ exclusive provider organization; $\mathrm{HDHP}=$ high-deductible health plan; $H M O=$ health maintenance organization; $P O S=$ point of service plan; $P P O=$ preferred provider organization; $S D=$ standard deviation . health care experiences. The MarketScan CCAE database was particularly useful for studying the costs of treatment for anal cancer because this disease affects many individuals who are aged younger than 65 years. CCAE data were collected from more than 100 large employers and health insurance plans for employees, early retirees, and Consolidated Omnibus Budget Reconciliation Act beneficiaries and their dependents who were covered by private insurance. Costs were measured for inpatient admissions, outpatient services, and drug prescriptions. The population in the CCAE represents more than 15\% of the employer-sponsored, privately insured, and noncapitated U.S. population younger than aged 65 years. In 2011, there were more than 50 million subjects in the CCAE database (49\% male and $51 \%$ female). Forty-one percent of subjects were aged $18-44$ years; $33 \%$ were aged $45-64$ years; and about $26 \%$ were aged younger than 18 years. Since the MarketScan data were de-identified, the study was exempt from institutional human subjects review.

\section{Case Identification}

The following International Classification of Disease, Ninth Revision, Clinical Modification (ICD-9-CM) codes were used to identify the anal cancer cases in the population: 154.2 (malignant neoplasm of digestive organs, peritoneum, and anal canal); 154.3 (malignant neoplasm of anus, unspecified site); and 154.8 (malignant neoplasm of other sites of rectum, rectosigmoid junction, and anus). We did not use the International Classification of Disease, Tenth Revision, Clinical Modification to identify the cases, since the diagnosis codes in the 2011-2014 MarketScan CCAE database were coded for ICD-9-CM.

Patients were included if they had at least 1 primary or secondary inpatient diagnosis claim for anal cancer or at least 2 primary or secondary outpatient diagnosis claims for anal cancer that were more than 30 days apart. The index date was defined as the first date an anal cancer diagnosis appeared in the data. Patients were excluded from the study if (a) they did not have continuous enrollment for 6 months before and after the index date, (b) they were diagnosed with cancer in any site (ICD-9-CM codes 140 through 208) during the 6 months before the index date, or (c) they were aged younger than 18 years.

We found 2 patients whose 2-year health care costs exceeded $\$ 1,000,000$ (\$2,087,635 in 1 patient and $\$ 1,129,032$ in the other). Because these costs were extremely high compared with other patients' costs, we excluded these 2 outlier patients to avoid overestimating the mean treatment cost.

\section{Control Selection}

Controls were selected from among patients in the MarketScan database without a diagnosis of anal cancer or other HPVrelated cancer (i.e., cervical cancer, oropharyngeal cancer, penile cancer, vulvar cancer, and vaginal cancer). Controls were randomly assigned index dates that matched case index 


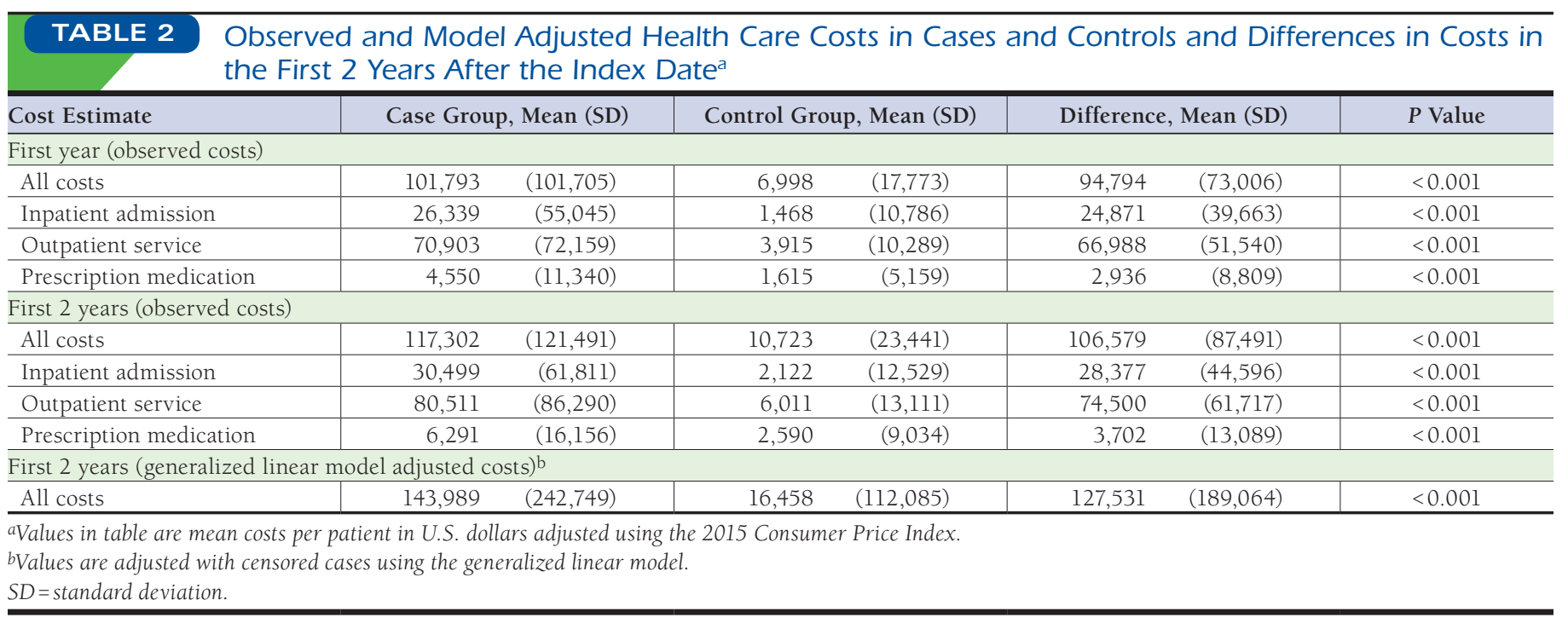

dates to permit health care cost assessment of controls and their matched cases during the same period. Controls were excluded if the patient did not have continuous enrollment for 6 months before and after the index date or if the patient was diagnosed with cancer in any site during the 6 months before the index date.

After application of the exclusion criteria, each case was matched with controls on the basis of (a) age \pm 5 years, (b) sex, (c) 9 geographic areas, and (d) index date. Propensity scores were computed for each case and candidate control for the following variables: (a) Charlson Comorbidity Index score during the 6 months before the index date, (b) psychiatric diagnostic group score during the 6 months before the index date, ${ }^{17}$ (c) prediagnosis costs, and (d) type of health insurance plan. The prediagnosis costs were defined as the health care costs during the first 3 months of the 6-month period before the index date. For example, if an individual's index date was July 1,2012 , the prediagnosis costs were the health care costs from January 1, 2012, through March 31, 2012. A propensity score and Mahalanobis distance were used to produce 1 to 1 case and control matching. ${ }^{18,19}$ Once a control was selected for a given case, the control was removed from consideration for additional matches.

\section{Study Outcomes}

Since the longest follow-up period in the study population was 3.5 years and the mean follow-up duration was approximately 20 months, we defined the primary study outcome as the per patient estimated 2-year health care costs for treatment of anal cancer. These costs were measured by calculating the difference in mean health care costs between the cases and controls in the first 2 years after the index date. A generalized linear model with log link function and gamma distribution was used for the cost estimates. Costs were adjusted for covariates including age, sex, having at least 1 year of follow-up or not, type of health insurance plan, geographic area, case/control status, Charlson Comorbidity Index score, psychiatric diagnostic group score, prediagnosis costs, 2 -years' month index from 1 to 24 , squared month index, the interaction terms between case/control status and month index, and the interaction terms between case/control status and squared month index.

The dependent variable for the model was the monthly health care costs during the 2-year follow-up period, which resulted in 24 partitions of estimated cost for each patient. To estimate the 2-year per patient health care costs, the censored data for the patients who did not have at least 2 years of follow-up were replaced with the model estimates. The monthly differences in health care costs between cases and controls allowed partitioning of costs between the initial treatment phase and the continuing phase of cancer treatment. ${ }^{20}$

Cost was defined as the total cost of copayments, coinsurance, deductibles, and coordination of benefits and other adjustments for inpatient admissions, outpatient services, and prescription medications. We included all the health care costs incurred, including insurer payments and patient outof-pocket payments. All costs were adjusted to year 2015 U.S. dollar values using the Consumer Price Index from the U.S. Bureau of Labor Statistics. ${ }^{21}$

\section{Results}

A total of 1,976 patients diagnosed with anal cancer during 2011-2014 were identified (Figure 1). The mean age of cases was 54 years, and there were more women than men $(63.82 \%$ vs. $36.18 \%$; Table 1). The majority of cases and controls were covered by a preferred provider organization. The South Atlantic region (Delaware, Maryland, Virginia, West Virginia, 
Health Care Costs of Anal Cancer in a Commercially Insured Population in the United States

\section{TABLE 3 Generalized Linear Model for Estimation of Per Patient Costs in the First 2 Years After the Index Date}

\begin{tabular}{|c|c|c|c|c|c|}
\hline Covariate & Estimated Coefficient & SE & \multicolumn{2}{|c|}{$95 \% \mathrm{CI}$} & $P$ Value \\
\hline Intercept & 5.90 & 0.32 & 5.26 & 6.53 & $<0.0001$ \\
\hline Age & 0.002 & 0.005 & -0.01 & 0.01 & 0.650 \\
\hline \multicolumn{6}{|l|}{ Sex } \\
\hline Female & -0.21 & 0.06 & -0.33 & -0.09 & 0.001 \\
\hline Male & Reference & & & & \\
\hline \multicolumn{6}{|l|}{ Health insurance plan type } \\
\hline EPO/unknown & 0.14 & 0.17 & -0.19 & 0.46 & 0.417 \\
\hline HMO/POS with capitation/POS & 0.22 & 0.13 & -0.04 & 0.47 & 0.092 \\
\hline $\mathrm{PPO}$ & 0.33 & 0.12 & 0.10 & 0.56 & 0.005 \\
\hline CDHP/HDHP & 0.29 & 0.17 & -0.05 & 0.62 & 0.094 \\
\hline Comprehensive & Reference & & & & \\
\hline \multicolumn{6}{|l|}{ Geographic area (division) } \\
\hline Middle Atlantic are & -0.01 & 0.15 & -0.32 & 0.29 & 0.930 \\
\hline East North Central area & 0.01 & 0.15 & -0.29 & 0.31 & 0.954 \\
\hline West North Central area & -0.35 & 0.17 & -0.67 & -0.02 & 0.036 \\
\hline South Atlantic area & 0.03 & 0.14 & -0.25 & 0.32 & 0.819 \\
\hline East South Central area & -0.04 & 0.18 & -0.39 & 0.31 & 0.806 \\
\hline West South Central area & -0.15 & 0.15 & -0.45 & 0.14 & 0.307 \\
\hline Mountain area & 0.07 & 0.18 & -0.29 & 0.43 & 0.711 \\
\hline Pacific area & -0.10 & 0.14 & -0.39 & 0.18 & 0.482 \\
\hline New England area & Reference & & & & \\
\hline \multicolumn{6}{|l|}{ Duration of follow-up } \\
\hline More than 1 year & -0.10 & 0.06 & -0.23 & 0.03 & 0.117 \\
\hline Less than or equal to 1 year & Reference & & & & \\
\hline \multicolumn{6}{|l|}{ Case/control } \\
\hline Case & 4.03 & 0.11 & 3.81 & 4.24 & $<0.001$ \\
\hline Control & Reference & & & & \\
\hline Charlson comorbidity index score & 0.18 & 0.06 & 0.06 & 0.29 & 0.003 \\
\hline Psychiatric diagnostic groups score & -0.53 & 0.14 & -0.80 & -0.26 & $<0.001$ \\
\hline Pre-diagnosis 3-month costs & 0.00005 & 0.000003 & 0.0 & 0.07 & $<0.001$ \\
\hline Month index & 0.03 & 0.02 & -0.01 & 0.06 & 0.181 \\
\hline Month index ${ }^{2}$ & -0.001 & 0.001 & -0.002 & 0.001 & 0.239 \\
\hline Month index $\times$ case & -0.29 & 0.02 & -0.34 & -0.25 & $<0.001$ \\
\hline Month index $\times$ control & Reference & & & & \\
\hline Month index ${ }^{2} \times$ case & 0.01 & 0.00 & 0.01 & 0.01 & $<0.001$ \\
\hline Month index ${ }^{2} \times$ control & Reference & & & & \\
\hline
\end{tabular}

$\mathrm{CDHP}=$ consumer-driven health plan; $C I=$ confidence interval; $E P O=$ exclusive provider organization; $H D H P=$ high-deductible health plan; HMO=health maintenance organization; $P O S=$ point of service plan; $P P O=$ preferred provider organization; $S E=$ standard error.

North Carolina, South Carolina, Georgia, Florida, and the District of Columbia) had the highest percentage of the patient population (20.14\%). Except for employment status and enrollment duration, there were no significant differences between cases and controls in other population characteristics.

The cost difference between cases and controls was $\$ 106,579$ (standard deviation $[S D]=\$ 87,491$ ) for the first 2 years after the index date without adjustment for covariates and censoring (Table 2). The greatest difference in costs between cases and controls in the first 2 years was the difference in costs of outpatient services (70\%), followed by inpatient admissions (27\%) and prescription drugs (3\%; Table 2). The top 3 highest cost items among the outpatient services were radiation treatment (per patient mean cost $=\$ 31,090[\mathrm{SD}=\$ 21,951], 14 \%$ of total outpatient costs); chemotherapy (per patient mean cost $=\$ 25,913$ [SD $=\$ 30,211]$, 4\% of total outpatient costs); and positron emission tomography with computed tomography (per patient mean cost $=\$ 4,810$ [SD $=\$ 4,756], 3 \%$ of outpatient costs), which is an alternative to standard computed tomography (data not shown).

After model adjustments for censored cases, the estimated total per patient health care cost for the first 2 years for patients with anal cancer was $\$ 127,531$ (Table 2). Sex, case/control status, Charlson Comorbidity Index score, psychiatric diagnostic groups score, prediagnosis costs, and interaction terms between case/control status and month index and between case/control status and squared month index were significantly associated 


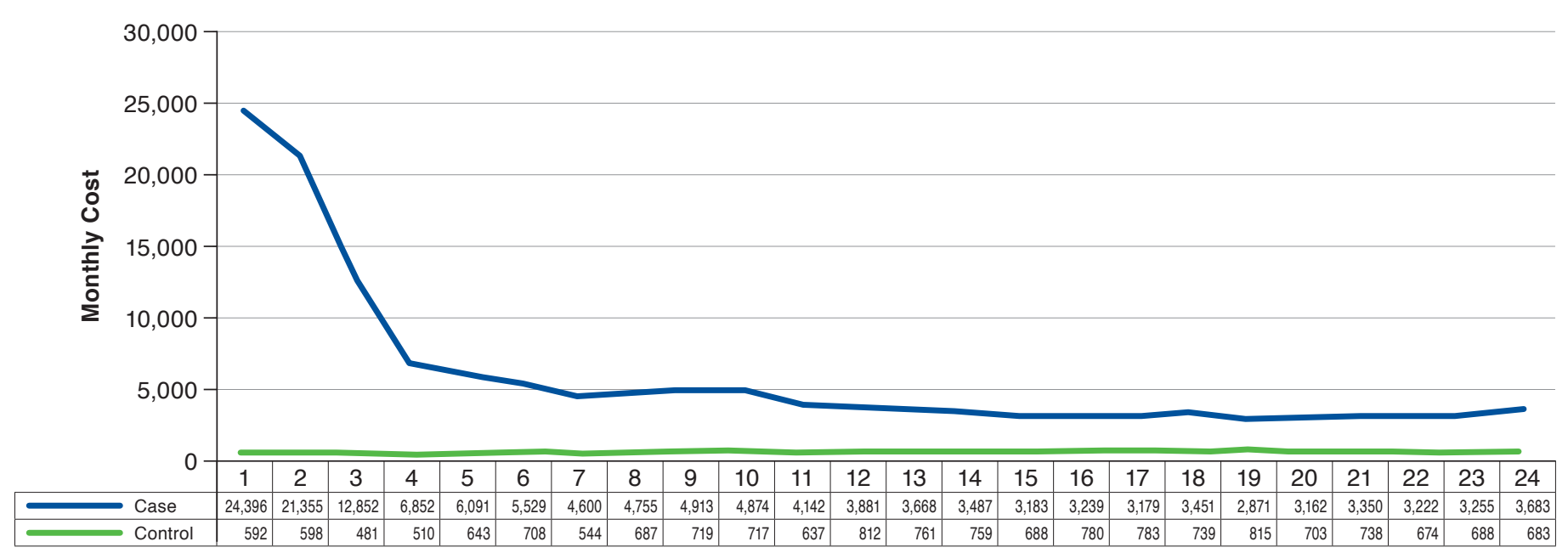

Month Index

with the 2-year health care costs (Table 3). The adjusted 2-year per patient health care costs for anal cancer patients were 8.7 times greater than the per patient costs for the matched noncancer patients. The case costs were highest in the first 6 months after diagnosis (per patient mean cost $=\$ 12,846$ ), leveling off at much lower monthly costs during the subsequent 18 months of the 2-year period (per patient mean cost $=\$ 3,713$; Figure 2). An example calculation of the adjusted cost using the model is provided in the Appendix (available in online article).

\section{Discussion}

By searching the 2011-2014 MarketScan CCAE database, we identified nearly 2,000 patients with anal cancer, with a mean age of 54 years. This mean age is in line with the 2010-2014 statistics of the SEER Program, ${ }^{22}$ which showed that the 10-year age group with the highest percentage of anal cancer cases diagnosed (29.4\%) comprised individuals aged 55-64 years. In our study population, the ratio of women to men was 1.5 to 1 . This gender distribution was similar to what has previously been reported in the literature, which indicated that anal cancer affects more women than men, showing a respective gender ratio of 1.4 to 1 in the U.S. population. ${ }^{2}$

We found that after adjustment for the non-normal cost distribution and censoring due to disenrollment, the estimated per patient treatment cost for anal cancer was $\$ 127,531$ in the first 2 years, or $\$ 63,766$ per year. The literature on anal cancer treatment cost for the privately insured population in the United States is limited. Hu and Goldie applied a Canadian costing study of rectal cancer to estimate the discounted lifetime per patient cost of anal cancer at $\$ 36,300$, adjusted to 2015 U.S. dollars. Because that study was based on the experience from a single-payer health care system using older medical technology, the total cost may significantly underestimate today's cost of treating anal cancer in the United States. ${ }^{9}$ Chesson et al. (2012) synthesized the results from $\mathrm{Hu}$ and Goldie and a previous HPV vaccine economic evaluation and then applied scenario analysis to estimate per patient anal cancer treatment cost at $\$ 39,800$, adjusted to 2015 U.S. dollars., ${ }^{93,24}$ In contrast, our study used real-world health care claims data with adjustments for covariates and non-normal cost distribution. Deshmukh et al. (2015) estimated the average lifetime cost per patient with anal cancer at \$51,200 (2015 U.S. dollars). ${ }^{12}$ However, since Deshmukh et al. focused on the Medicare-insured population, their results are not directly comparable to those of the present study.

Male sex, cancer diagnosis, higher Charlson Comorbidity Index score, and higher prediagnosis costs were significantly associated with higher per patient monthly costs in our study. An unexpected result was that higher psychiatric diagnostic group scores were associated with lower per patient monthly costs. While psychiatric conditions may present an access to care barrier, this could not be determined from the claims data.

Most of the cost in our population was for outpatient services (70\%), followed by inpatient admissions (27\%) and prescription drugs (3\%). The outpatient service with the highest cost was radiation treatment, with a mean cost of $\$ 12,000$ per patient. Patients incurred the highest costs in the first 6 months after the index date. After the sixth month, the cost pattern remained stable until the end of follow-up. This cost pattern matches what we expected in terms of anal cancer 
treatment. After diagnosis, the primary treatments for patients were combined chemotherapy and radiation. Those treatments accounted for the bulk of the cost. Treatment duration varied by the individuals who experience differences in disease progression. However, high costs in the first 6 months were also observed for other HPV-related cancer. ${ }^{25-27}$ Following the primary treatments, regular examination (e.g., clinic visits or anoscopy) was provided, which resulted in stable costs during the continuing care phase of treatment. Deshmukh et al. defined the first 6 months after diagnosis as the initial phase and the last 12 months before death as the terminal phase and found that patients with anal cancer had the highest costs in the initial and terminal phases..$^{12}$ Our future studies will focus on estimating the lifetime cost to provide a more complete estimate of the treatment cost for anal cancer.

Much of the cost and pain and suffering associated with anal cancer and other HPV-related cancers can be prevented by expanding the low HPV vaccination rates in the United States. Out-of-pocket cost is not a barrier because under the Affordable Care Act, HPV vaccination was assessed to be highly effective and cost-effective and, therefore, must be fully covered by qualified health insurance programs. ${ }^{28}$ The vaccine is also covered fully by the Vaccines for Children Program. ${ }^{29}$ Community-based pharmacies along with primary schools (through twelfth grade) have been identified as the most promising alternative sites for expanding the vaccination rates due to their convenience, experience with vaccinations, and generally high trust among the population. ${ }^{6}$ These alternative sites are especially good for adolescent boys who make relatively few visits to primary care physicians. Ideally, pharmacists will collaborate with primary care physicians to educate and encourage parents to vaccinate their children in the most convenient and cost-effective settings.

\section{Limitations}

This study has a number of limitations. First, MarketScan CCAE data covered the population aged younger than 65 years that was insured by private insurance plans. Therefore, the results do not generalize to populations aged 65 years or older or people covered by Medicare or Medicaid. Second, since the death information in the MarketScan database was not comprehensive, an estimate of the per patient cost for the terminal phase of illness was not feasible. Third, cancer stage was not available in the database, which precluded analysis of cost by stage at diagnosis. Fourth, data from anal adenocarcinoma may skew the results. However, information on pathology confirmation to differentiate squamous cell carcinoma and anal adenocarcinoma was not available in the database.

Despite these limitations, this study used real-world claims payment data, which are broadly representative of the U.S. privately insured population.

\section{Conclusions}

The first 2-year per patient cost associated with anal cancer treatment was $\$ 127,531$. Anal cancer treatment costs were highest in the first 6 months after diagnosis (mean PPPM $=\$ 12,846$, median PPPM $=\$ 3,188$ ), leveling off at much lower costs during the subsequent 18 months of the 2-year period (mean PPPM $=\$ 3,717$, median PPPM $=\$ 1,756)$. With about $79 \%$ of anal cancers attributable to HPV infection, this study provides important parameters for estimating the potential economic benefit of HPV vaccination.

\section{Authors}

CHI-FANG WU, MS, and DAVID R. LAIRSON, PhD, Department of Management, Policy, and Community Health, School of Public Health, The University of Texas Health Science Center at Houston. LI XU, PhD, Department of Head and Neck Surgery, Division of Surgery, and CRAIG A. MESSICK, MD, FACS, FASCRS, Department of Surgical Oncology, Section of Colon and Rectal Surgery, The University of Texas MD Anderson Cancer Center, Houston. SHUANGSHUANG FU, MS, Department of Epidemiology, Human Genetics, and Environmental Sciences, School of Public Health, and HO-LAN PENG, PhD, Department of Biostatistics, School of Public Health, The University of Texas Health Science Center at Houston.

AUTHOR CORRESPONDENCE: David R. Lairson, PhD, Department of Management, Policy, and Community Health, School of Public Health, The University of Texas Health Science Center at Houston, 7000 Fannin, Ste. 1200, Houston, TX 77030 Tel.: 713.500.9176; E-mail: David.R.Lairson@uth.tmc.edu.

\section{DISCLOSURES}

This research was accomplished within the Oropharynx Program at The University of Texas MD Anderson Cancer Center and was funded in part through the Stiefel Oropharyngeal Research Fund. The authors report funding contributions from the Christopher and Susan Damico Chair in Viral Associated Malignancies (The University of Texas MD Anderson). This work was supported by generous philanthropic contributions, including a contribution from the Lyda Hill Foundation, to The University of Texas MD Anderson HPV-Related Cancers Moon Shot Program. The authors have nothing to disclose.

\section{ACKNOWLEDGMENTS}

The authors thank Stephanie Deming for assisting with reviewing and editing the manuscript.

\section{REFERENCES}

1. Siegel RL, Miller KD, Jemal A. Cancer statistics, 2017. CA Cancer J Clin. 2017;67(1):7-30

2. National Cancer Institute. Surveillance, Epidemiology, and End Results Program. SEER cancer statistics review (CSR) 1975-2014. April 2017. Available at: https://seer.cancer.gov/archive/csr/1975_2014/. Accessed August 3, 2018, 
3. Saraiya M, Unger ER, Thompson TD, et al. U.S. assessment of HPV types in cancers: implications for current and 9-valent HPV vaccines. J Natl Cancer Inst. 2015;107(6): dvj086.

4. Shiels MS, Pfeiffer RM, Chaturvedi AK, Kreimer AR, Engels EA. Impact of the HIV epidemic on the incidence rates of anal cancer in the United States. J Natl Cancer Inst. 2012;104(20):1591-98.

5. Machalek DA, Poynten M, Jin F, et al. Anal human papillomavirus infection and associated neoplastic lesions in men who have sex with men: a systematic review and meta-analysis. Lancet Oncol. 2012;13(5):487-500.

6. Shah PD, Gilkey MB, Pepper JK, Gottlieb SL, Brewer NT. Promising alternative settings for HPV vaccination of U.S. adolescents. Expert Rev Vaccines. 2014;13(2):235-46.

7. Brisson M, Laprise JF, Chesson HW, et al. Health and economic impact of switching from a 4-valent to a 9-valent HPV vaccination program in the United States. J Natl Cancer Inst. 2016;108(1):djv282.

8. Elbasha EH, Dasbach EJ. Impact of vaccinating boys and men against HPV in the United States. Vaccine. 2010;28(42):6858-67.

9. Hu D, Goldie S. The economic burden of noncervical human papillomavirus disease in the United States. Am J Obstet Gynecol. 2008;198(5):500.el-7.

10. Maroun J, Ng E, Berthelot JM, et al. Lifetime costs of colon and rectal cancer management in Canada. Chronic Dis Can. 2003;24(4):91-101.

11. Goldie SJ, Kuntz KM, Weinstein MC, Freedberg KA, Palefsky JM. Costeffectiveness of screening for anal squamous intraepithelial lesions and anal cancer in human immunodeficiency virus-negative homosexual and bisexual men. Am J Med. 2000;108(8):634-41.

12. Deshmukh AA, Zhao H, Franzini L, et al. Total lifetime and cancerrelated costs for elderly patients diagnosed with anal cancer in the United States. Am J Clin Oncol. 2018;41(2):121-27.

13. Olsen J, Jørgensen TR, Kofoed K, Larsen HK. Incidence and cost of anal, penile, vaginal and vulvar cancer in Denmark. BMC Public Health. 2012;12:1082.

14. Office of Disease Prevention and Health Promotion, Healthy People 2020. Increase the vaccination coverage level of 3 doses of human papillomavirus (HPV) vaccine for females by age 13 to 15 years. 2018. Available at: https://www.healthypeople.gov/node/4657/data_details. Accessed August 3, 2018.

15. Reagan-Steiner S, Yankey D, Jeyarajah J, et al. National, regional, state, and selected local area vaccination coverage among adolescents aged 13-17 yearsUnited States, 2015. MMWR Morb Mortal Wkly Rep. 2016;65(33):850-58.
16. Bruni L, Diaz M, Barrionuevo-Rosas L, et al. Global estimates of human papillomavirus vaccination coverage by region and income level: a pooled analysis. Lancet Glob Health. 2016;4(7):e453-63.

17. Ashcraft ML, Fries BE, Nerenz DR, et al. A psychiatric patient classification system: an alternative to diagnosis-related groups. Med Care. 1989;27(5):543-57.

18. Rubin DB. Bias reduction using Mahalanobis-metric matching. Biometrics. 1980;36(2):293-98.

19. Austin PC. A comparison of 12 algorithms for matching on the propensity score. Stat Med. 2014;33(6):1057-69.

20. Brown ML, Riley GF, Schussler N, Etzioni R. Estimating health care costs related to cancer treatment from SEER-Medicare data. Med Care. 2002;40(8 Suppl):IV-104-17.

21. Bureau of Labor Statistics. Consumer Price Index, medical care. 2015. Available at: http://data.bls.gov/cgi-bin/surveymost?cu. Accessed August 3, 2018.

22. National Cancer Institute. Surveillance, Epidemiology, and End Results Program (SEER). Cancer stat facts: anal cancer. Available at: https://seer.cancer.gov/statfacts/html/anus.html. Accessed August 3, 2018.

23. Chesson HW, Ekwueme DU, Saraiya M, Watson M, Lowy DR, Markowitz LE. Estimates of the annual direct medical costs of the prevention and treatment of disease associated with human papillomavirus in the United States. Vaccine. 2012;30(42):6016-19.

24. Kim JJ, Goldie SJ. Health and economic implications of HPV vaccination in the United States. N Engl J Med. 2008;359(8):821-32.

25. Fu S, Lairson DR, Chan W, Wu CF, Ramondetta L. Mean medical costs associated with vaginal and vulvar cancers for commercially insured patients in the United States and Texas. Gynecol Oncol. 2018;148(2):342-48.

26. Lairson DR, Fu S, Chan W, Xu L, Shelal Z, Ramondetta L. Mean direct medical care costs associated with cervical cancer for commercially insured patients in Texas. Gynecol Oncol. 2017;145(1):108-13.

27. Lairson DR, Wu CF, Chan W, Dahlstrom KR, Tam S, Sturgis EM. Medical care cost of oropharyngeal cancer among Texas patients. Cancer Epidemiol Biomarkers Prev. 2017;26(9):1443-49.

28. Henry J. Kaiser Family Foundation. The HPV vaccine: access and use in the U.S. 2017. Available at: https://www.kff.org/womens-health-policy/factsheet/the-hpv-vaccine-access-and-use-in/. Accessed August 3, 2018.

29. American Cancer Society. HPV vaccines. Available at: https://www. cancer.org/cancer/cancer-causes/infectious-agents/hpv/hpv-vaccines.html. Accessed August 3, 2018. 


\section{APPENDIX An Example of How to Determine the Adjusted Per Patient Cost Following the Study Regression} Model with Log Link Function

Based on the study model, the estimated function for the 2-year cost for the study population was determined according to the following calculations:

$\operatorname{Ln}($ cost $)=5.90+(0.002 \times$ Age $)-(0.21 \times$ Female $)+(0.14 \times$ EPO/unknown $)+(0.22 \times$ HMO/POS plan with capitation/POS plan $)+(0.33 \times \mathrm{PPO})+$

$(0.29 \times$ CDHP/HDHP $)-(0.01 \times$ Middle Atlantic division $)+(0.01 \times$ East North Central division $)-(0.35 \times$ West North Central division $)+(0.03 \times$ South Atlantic division) - (0.04 $\times$ East South Central division) $-(0.15 \times$ West South Central division $)+(0.07 \times$ Mountain division $)-(0.10 \times$ Pacific

division $)-(0.10 \times$ More than 1 year follow - up $)+(4.03 \times$ Case $)+(0.18 \times$ Charlson Comorbidity Index score $)-(0.53 \times$ Psychiatric diagnostic group score $)+(0.00005 \times$ Prediagnosis 3 - month costs $)+(0.03 \times$ Month index $)-\left(0.001 \times\right.$ Month index $\left.{ }^{2}\right)-(0.29 \times$ Interaction term between month index and case/control status $)+\left(0.01 \times\right.$ Interaction term between month inde ${ }^{2}$ and case/control status $)$.

The estimated first month cost in the case of a man aged 61 years, who was a resident of the New England division with less than 1 year of follow-up, was calculated using the following additional covariate data: coverage by an HMO/POS plan with capitation/POS plan, Charlson Comorbidity Index score of 1, psychiatric diagnostic group score of zero, and prediagnosis 3 -month costs of $\$ 21,854$. The calculations are shown below:

$$
\operatorname{Exp}[5.90+(61 \times 0.002)+0.22+4.03+(1 \times 0.18)+(21,548 \times 0.00005)+(0.03 \times 1)-(0.001 \times 1)-0.29+0.01]=\operatorname{Exp}(11.2784)=79,095 .
$$

Calclulations for the estimated second month's cost are shown below:

$$
\operatorname{Exp}[5.90+(61 \times 0.002)+0.22+4.03+(1 \times 0.18)+(21,548 \times 0.00005)+(0.03 \times 2)-(0.001 \times 2 \times 2)-(0.29 \times 2)+(0.01 \times 2 \times 2)]=\operatorname{Exp}(11.0454)=62,655
$$

